\title{
Core-Scale Description of Porous Media Dissolution During Acid Injection - Part II: Calculation of the Effective Properties
}

\author{
FABRICE GOLFIER ${ }^{1}$, MICHEL QUINTARD ${ }^{2}$, BRIGITTE BAZIN ${ }^{3}$ \\ and ROLAND LENORMAND ${ }^{3}$ \\ ${ }^{1}$ Laboratoire Environnement, Géomécanique et Ouvrages, LAEGO, ENSG, INPL \\ Rue du Doyen Marcel Roubault, BP40, 54501 Vandoeuvre-lès-Nancy, France \\ ${ }^{2}$ Institut de Mécanique des Fluides, Allée C. Soula, 31400 Toulouse, France \\ ${ }^{3}$ IFP, Avenue de Bois-Préau, 92852 Rueil-Malmaison Cedex, France \\ Email: fabrice.golfier@ensg.inpl-nancy.fr
}

\begin{abstract}
Acid injection in porous medium is a process widely used for stimulation of petroleum wells and leads to the formation of highly conductive channels called wormholes. Two different transport-reaction models have been developed in Part I to describe the phenomenon at the core-scale. The possible existence of core-scale effective properties which appear in these models is discussed here on the basis of Darcy-scale numerical experiments. The advantages and drawbacks of one-equation and two-equation models are investigated by reference to averaged fields computed from Darcy-scale simulations.
\end{abstract}

Mathematical subject classification: Primary 74Q20; Secondary 80A32.

Key words: core-scale dissolution, acidification, effective properties, porous media, wormhole.

\section{Introduction}

The unstable dissolution of a porous medium leads to complex patterns which are difficult to model quantitatively [4]. Indeed, this dissolution process is coupled with the fluid momentum equation in an unstable way: flow velocity is higher in the largest pores, which generally produces faster dissolution processes. These

\#605/04. Received: 07/X/04. Accepted: 15/XII/05. 
processes increase locally the pore diameter and this may in turn facilitate the acid transport to these large pores. These physical mechanisms can lead to the formation of highly conductive flow channels called wormholes. A model has been developed at the Darcy-scale by Golfier et al. [1] involving a local nonequilibrium dissolution equation. Numerical simulations have been performed for 2D and 3D configurations for both homogeneous and heterogeneous systems and allowed to capture all the observed features in terms of dissolution regimes and optimum flow rate. However, a direct application of laboratory results to the field scale is not straightforward since a direct Darcy-scale description would require a very fine grid. Therefore, a large-scale model is necessary.

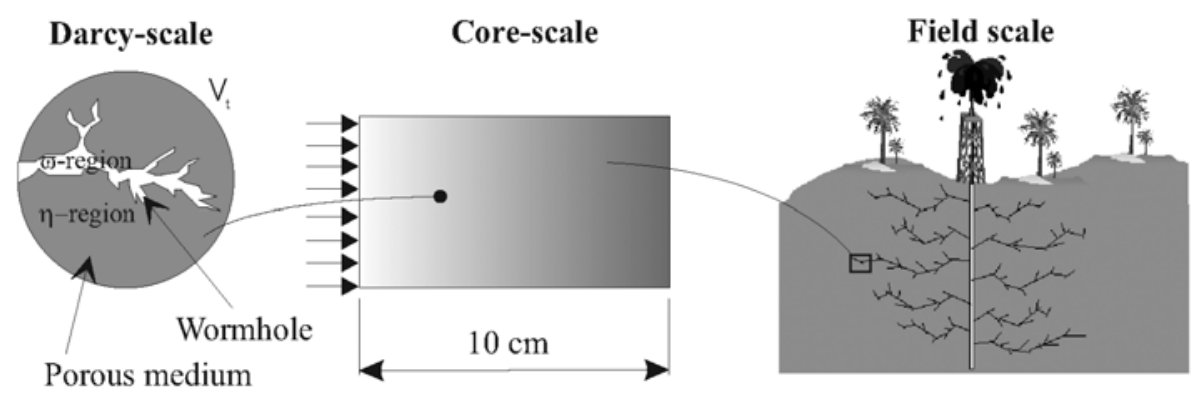

Figure 1 - The different scales of the problem.

A first attempt has been made in Part I [2] at deriving a core-scale dissolution model based on cross-sectional averages from a volume averaging method $[5,10]$. We remind below the notations used at the core-scale and briefly summarize the different approaches developed in Part I [2]. It must be emphasized here that this study does not concern only stimulation methods by acidification of petroleum wells but the dissolution mechanisms in porous media in a more general way. As a consequence, we consider only the interaction of the wateracid mixture with the rock (single-phase flow) and the oil phase is not taken into account. Core-scale averaged quantities are defined in the core-scale averaging volume illustrated in Fig. 1. For example, the regional intrinsic acid concentration and the superficial regional velocity associated to the $\varpi-$ region, 
respectively $C_{A \varpi}^{*}$ and $\mathbf{V}_{\varpi}^{*}$, are defined as follows.

$$
\begin{gathered}
C_{A \varpi}^{*}=\left\{C_{A \beta}\right\}_{\varpi}^{\varpi}=\frac{1}{V_{\varpi}} \int_{V_{\varpi}} C_{A \beta} d V \\
\mathbf{V}_{\varpi}^{*}=\left\{\mathbf{V}_{\beta}\right\}_{\varpi}=\frac{1}{V_{t}} \int_{V_{\varpi}} \mathbf{V}_{\beta} d V
\end{gathered}
$$

Flow equation. For the flow description at this scale, a classical Darcy's law has been obtained by averaging the Darcy-Brinkman formulation.

$$
\begin{gathered}
\mathbf{V}_{\beta}^{*}=-\frac{1}{\mu_{\beta}} \mathbf{K}^{*} \cdot\left(\nabla P_{\beta}^{*}-\rho_{\beta} \mathbf{g}\right) \\
\nabla \cdot \mathbf{V}_{\beta}^{*}=0
\end{gathered}
$$

where $\mathbf{V}_{\beta}^{*}$ and $P_{\beta}^{*}$ represent respectively the core-scale averaged velocity and pressure and $\mathbf{K}^{*}$ the core-scale permeability tensor. It must be pointed out that this average velocity is linked to the two regional average velocities $\mathbf{V}_{\varpi}^{*}$ and $\mathbf{V}_{\eta}^{*}$, with the following relation:

$$
\mathbf{V}_{\beta}^{*}=\mathbf{V}_{\varpi}^{*}+\mathbf{V}_{\eta}^{*}
$$

With regard to the transport and dissolution part, it was not obvious to apply an upscaling method which would lead to some core-scale equations valid in a general way. In fact, the value of the mass transfer coefficient appearing in the Darcy-scale model can strongly modify the acid transport behavior and the corresponding Darcy-scale dissolution pattern. Several approaches have been developed depending on whether the local mass equilibrium condition is verified or not.

Local mass equilibrium dissolution: Two-equation model. If the value of the mass transfer coefficient is very large, we obtain two different regions at the Darcy-scale: a fluid region in the dissolved areas, and a porous region where dissolution has not occurred. This looks like a double-porosity system, and this suggests the introduction of a two-equation model for which the wormholes 
( $\varpi-$ region) and the remaining porous matrix ( $\eta$-region) are treated separately. The transport equations for the $\varpi-$ region are written as

$$
\begin{gathered}
\phi_{\varpi} \frac{\partial C_{A \varpi}^{*}}{\partial t}+\mathbf{V}_{\varpi}^{*} \cdot \nabla C_{A \varpi}^{*}=\nabla \cdot\left(\mathbf{D}_{\varpi}^{* *} \cdot \nabla C_{A \varpi}^{*}\right)-\alpha^{*} C_{A \varpi}^{*} \\
\frac{\partial \phi_{\varpi}}{\partial t}=\frac{\beta_{s}}{\rho_{\sigma}} \alpha^{*} C_{A \varpi}^{*}
\end{gathered}
$$

where $\beta_{s}$ represents the stoichiometric coefficient of the reaction, $\rho_{\sigma}$ the rock density, $\alpha^{*}$ the core-scale mass transfer coefficient and $\phi_{\varpi}$ the wormhole volume fraction whereas the average concentration associated to the $\eta$-region, $C_{A \eta}^{*}$, is equal to 0 .

Local mass non-equilibrium dissolution: One-equation model. In the more general case of local non-equilibrium conditions at the Darcy-scale, the more complex form of the local equations did not allow to directly infer the core-scale transport equation. Nevertheless, it was possible to propose a general form for the macroscopic transport-reaction equations within a one-equation model [6, 9], defined as follows:

$$
\begin{aligned}
\varepsilon_{\beta}^{*} \frac{\partial C_{A \beta}^{*}}{\partial t}+\mathbf{V}_{\beta}^{*} \cdot \nabla C_{A \beta}^{*} & =\nabla \cdot\left(\mathbf{D}_{\beta}^{* *} \cdot \nabla C_{A \beta}^{*}\right)-\alpha^{*} C_{A \beta}^{*} \\
\frac{\partial \varepsilon_{\beta}^{*}}{\partial t} & =\frac{\beta_{s}}{\rho_{\sigma}} \alpha^{*} C_{A \beta}^{*}
\end{aligned}
$$

where $\varepsilon_{\beta}^{*}$ represents the core-scale porosity and $C_{A \beta}^{*}$ the average concentration weighted by the porosity.

While we have now decided on the form of the core-scale equations to be used in our model, a fundamental question remains: how to determine the different effective coefficients that appear in these core-scale equations? They could be calculated in principle by solving so-called closure problems. However, this requires the knowledge of the developed wormhole geometry, which is the result of the flow process. As a consequence of the fluid-porous medium interface evolution during the dissolution process, the relationship between the macroscopic quantities may be historical. This problem exists also for the Darcy-scale model, and we have adopted in this case the assumption classically made in geochemistry of a direct relationships linking the different macroscopic properties. 
For instance, permeability is a function of porosity, or the mass transfer coefficient depends on the cell Peclet number and porosity. Is it possible to use some similar relations at the core-scale?

In order to test such a possibility, rather than to solve numerically the closure problems with some non-representative boundary conditions, the dissolution patterns obtained at the Darcy-scale are used in this work to determine the effective coefficients. A series of Darcy-scale simulation is performed on a core of 25 $\mathrm{cm}$ length and $5 \mathrm{~cm}$ wide and used to obtain, by spatial integration, some corescale effective parameters. We remind briefly in a first part the various results extracted from Darcy-scale simulations and presented in Golfier et al. [1]. Then, the definitions associated with the introduction of the core-scale parameters are presented and the possible independence of these coefficients with respect to the dissolution history is discussed based on the numerical experiments.

\section{Darcy-scale numerical simulations}

Different Darcy-scale simulations have been performed on a two-dimensional domain by varying the injection flow rate $Q$, the initial acid concentration $C_{0}$ and the mass transfer coefficient value $\alpha$. Two principal results have been extracted from the model. First, the simulations allow to capture the different dissolution regimes, those corresponding to local equilibrium as well as those with nonequilibrium. We can see in Fig. 2 from Golfier et al. [1] an example of the different dissolution figures obtained numerically for different acid injection rates. All these figures represent the porosity field. The obtained dissolution patterns are remarkably equivalent to the experimental dissolution patterns. These results were used to draw diagrams of the transitions between the different regimes.

Secondly, the performed simulations have confirmed the existence of an optimum injection rate. This numerical optimum injection rate has been fitted with the experimental value through the proper choice of a single parameter, $A$, defining the mass transfer coefficient value through

$$
\alpha\left(P e_{\text {cell }}, \varepsilon_{\beta}\right)=A \alpha_{0}\left(P e_{\text {cell }}, \varepsilon_{\beta}\right)
$$

where $\alpha_{0}$ is the correlation obtained from the "closure problem", presented in Golfier et al. [1]. This latter correlation expresses the mass transfer coefficient 
(a) (b)

(c)

(d)

(e)

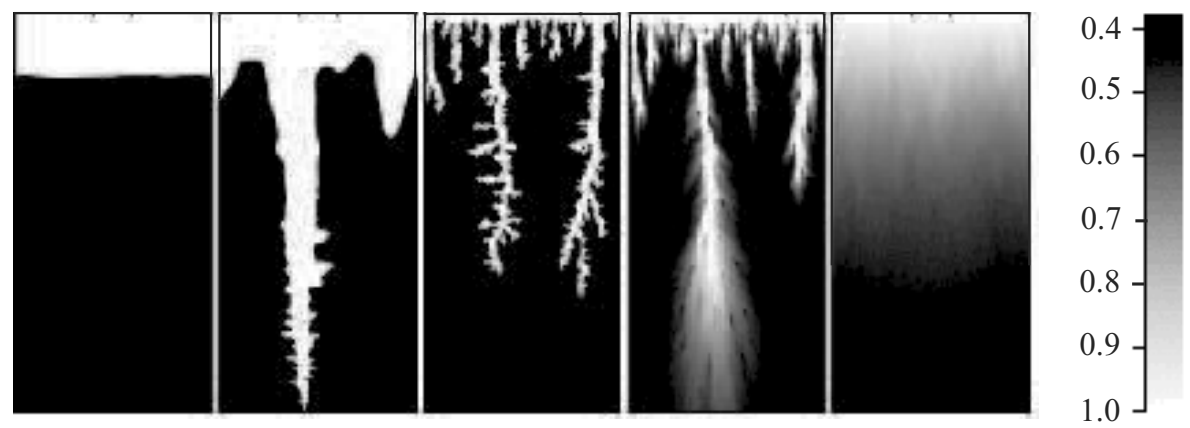

Figure 2-Porosity fields representative of the dissolution structure obtained numerically: Example of dissolution patterns: (a) face dissolution, $P e=8.32 \times 10^{-4}, D a=120$; (b) conical wormhole, $P e=4.14 \times 10^{-3} \%, D a=24$; (c) dominant wormhole, $P e=1.66$, $D a=6.01 \times 10^{-2}$; (d) ramified wormhole, $P e=83.2, D a=1.2 \times 10^{-3}$; (e) uniform dissolution, $P e=832, D a=1.2 \times 10^{-4}$ (Golfier et al., J. Fluid Mech., 457, 2002).

as a function of the Peclet number, defined at the Darcy-scale $P e_{c e l l}$, and the porosity $\varepsilon_{\beta}$.

The calculations show that the optimum injection rate is linked with the Damköhler number value, called $D a^{1}$. Also, this model allowed to study the effect of many external parameters, such as the variation of temperature or concentration, or the use of other reacting fluids, like acids in emulsion, which give lower diffusion effects.

\section{Macroscopic parameters and core-scale relations}

In this section, we discuss the determination of correlations between the different core-scale effective coefficients from numerical experiments performed at the Darcy-scale. The definitions associated with these core-scale parameters are discussed below. We consider the domain represented in Fig. 1. Averaged properties, that could be used in a 1D core-scale representation are defined as follows. The different parameters of the problem (pressure, velocity, concentration...) are integrated on some interval of given length (Fig. 3) to provide

\footnotetext{
${ }^{1}$ The Damköhler number is a dimensionless number, defined as the ratio between the acid consumed and the acid transported by convection.
} 
the core-scale properties, whose definitions have been given in Part I [2] and reminded in the introduction.

From the resulting porosity, pressure, velocity, and concentration fields, two additionnal core-scale parameters are calculated. From the pressure and velocity fields, we calculate the head loss between two sections of the core. From the concentration field, we calculate the overall dissolution rate for a volume included between two cross sections. From these parameters it is possible to determine the core-scale permeability and the mass exchange coefficient. It must be emphasized that their definition is general, and does not depend on a particular model.

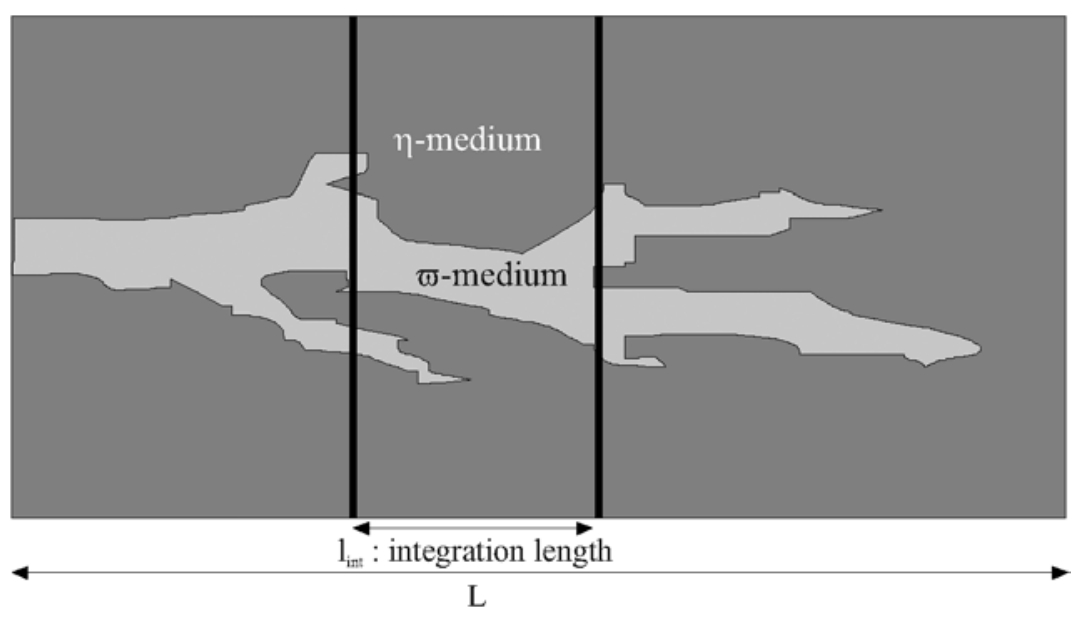

Figure 3 - Core-scale approach of the problem.

- Core-scale averaged permeability:

$$
K^{*}=\mu_{\beta}\left(\frac{\partial P_{\beta}^{*}}{\partial x}\right)^{-1}\left(\mathbf{V}_{\beta}^{*}\right)_{x}
$$

- Core-scale averaged mass transfer coefficient:

$$
\alpha^{*}=\frac{1}{C_{A \beta}^{*}} \frac{1}{V_{t}} \int_{V_{t}} \alpha C_{A \beta} d A
$$

These different parameters can be calculated as a function of time for the various performed numerical experiments. What is the impact of the dissolution 
history? In fact, as mentioned in the introduction, a direct relationship between the different macroscopic quantities may be historical, due to the coupling between the flow velocity and the dissolution process. It must be emphasized that this question is of a general interest, and similar problems were found in geochemistry, or in dealing with dendritic mushy zones [7, 3]. This problem has been solved approximately at the Darcy-scale, where we have already determined the relations linking the different macroscopic properties, such as the permeability versus the porosity or the mass transfer coefficient as a function of the cell Peclet number and the porosity. We discuss below the application of the same ideas at the upper scale, by focusing particularly on the relations $\mathbf{K}^{*}-\phi_{\varpi}$ and $\alpha^{*}=f\left(P e, \phi_{\varpi}, \ldots\right)$.

\subsection{Macroscopic permeability relationship}

We begin by representing the evolution of the core-scale permeability $\mathbf{K}^{*}$ as a function of the fluid fraction $\phi_{\varpi}$, or the core-scale porosity $\varepsilon_{\beta}^{*}$, for the dissolution regimes: conical wormhole, wormholing and ramified wormhole. The reason for keeping the two parameters $\phi_{\varpi}$ and $\varepsilon_{\beta}^{*}$ in the analysis have been explained in Part I [2]. In the case of local equilibrium, the Darcy-scale porosity remains constant in the non-dissolved areas. Thus, the single parameter characteristic of dissolution is $\phi_{\varpi}$. On the contrary, in the non-equilibrium cases, the Darcy-scale porosity varies, and the two parameters $\phi_{\bar{\sigma}}$ and $\varepsilon_{\beta}^{*}$ characterize the dissolution process, in a somehow independent manner.

For the two limit cases of dissolution, compact and uniform regime, the problem simplifies to a 1D case and the dissolution front velocity is constant. It is remarkable to see that local equilibrium as well as non-equilibrium dissolution can lead to a stationary front displacement. Given the displacement velocity of the front is constant, the $\mathbf{K}^{*}-\phi_{\varpi}$ relation does not depend on the time evolution although it is still difficult to express easily this relationship, especially under local non-equilibrium conditions because of the Darcy-scale porosity gradients.

For the three other cases named above, the question remains open. We tried to determine a relation between core-scale permeability and porosity in a similar way to the Darcy-scale problem. 


\subsubsection{Compact regime}

For this regime, we have a sharp dissolution front with a displacement velocity $\mathbf{V}_{q}$ equal to

$$
\mathbf{V}_{q}=\frac{\mathbf{V}_{0} C_{0}}{\frac{\rho_{\sigma} \varepsilon_{\sigma}}{\beta}+C_{0}}
$$

where $\mathbf{V}_{0}$ and $C_{0}$ are respectively the injection velocity and injection concentration.

The core-scale permeability field $K^{*}$ is directly linked to the core-scale volume fraction $\phi_{\varpi}$, and their values depend on the position of the cross section versus the dissolution front. We can write the relationship $K^{*}-\phi_{\varpi}$, for $0 \leq \phi_{\varpi} \leq 1$, under the form

$$
K^{*}=\frac{K K_{\text {fluid }}}{\left(1-\phi_{\varpi}\right) K_{\text {fluid }}+\phi_{\varpi} K}
$$

where $K_{\text {fluid }}$ represents the equivalent permeability of the fluid region.

\subsubsection{Conical regime}

Although the effective coefficients $K^{*}(t)$ and $\phi_{\varpi}(t)$ are a function of time, their evolutions, nevertheless, are not independent. Is it possible to obtain a relationship between these two parameters which is independent of the dissolution history or does it stay a function of time?

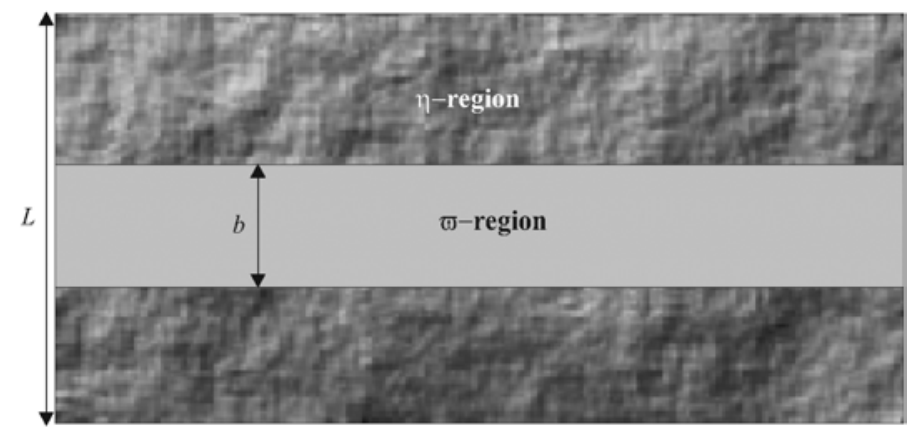

Figure 4 - Schematic representation of tube dissolution.

To answer this question, let us focus on a simplified case of our problem. We consider a wormhole which propagates into a porous medium of initial permeability $K_{0}$ and of width $L$. The studied part of the domain is far enough from 
the boundaries so that the flow may be considered steady (see Fig. 4). If we represent the wormhole by a tube of width $b$, the effective permeability $K^{*}$ can be estimated analytically. The equivalent permeability for the fluid zone is on the order of $b^{2} / 12$ (in 2D) which leads, for the permeability $K^{*}$, to

$$
\begin{aligned}
K^{*} & =\left(1-\phi_{\varpi}\right) K_{0}+\phi_{\varpi} b^{2} / 12 \\
& \approx \frac{\phi_{\varpi} b^{2}}{12} \text { for } \phi_{\varpi}>0
\end{aligned}
$$

and the channel width $b$ can be also expressed as a function of the fluid fraction, i.e. $b=\phi_{\varpi} L$. We obtain:

$$
K^{*}=\frac{L^{2} \phi_{\varpi}^{3}}{12}
$$

This relationship gives us an idea about the $\mathbf{K}^{*}-\phi_{\varpi}$ correlation existing in our problem.

However, we are generally far from these theoretical conditions. We have represented in Fig. 5 the macroscopic permeability as a function of the fluid fraction at $Q=1 \mathrm{~cm}^{3} \cdot \mathrm{h}^{-1}$ for different dissolution times. The integration interval is fixed at $l_{\text {int }}=0.75 \mathrm{~cm}$. It is clear from these results that the permeability evolution remains a function of the time dissolution, except for small volume fractions $\phi_{\varpi}$, and we have

$$
K^{*}=f\left(\phi_{\varpi}, t\right)
$$

This result can be physically explained from the dissolution figures. The closest we are from the inlet of the domain (i.e., the bigger the fluid fraction is), the less the wormhole can be represented by a tube in the studied section and, consequently, Eq. (16) is no longer verified.

\subsubsection{Wormholing regime}

On the contrary, for the wormholing regime, the obtained results are closer to those predicted by the simplified theoretical model developed as indicated above. In fact, in this case, the wormhole geometry is similar to a tube. We have represented in Fig. 6, the $K^{*}-\phi_{\varpi}$ relation for different injection rates, different values of the mass transfer coefficient $\alpha$ and at different dissolution times. The interval length $l_{\text {int }}$ is also equal to $0.75 \mathrm{~cm}$. We see that, for this dissolution 


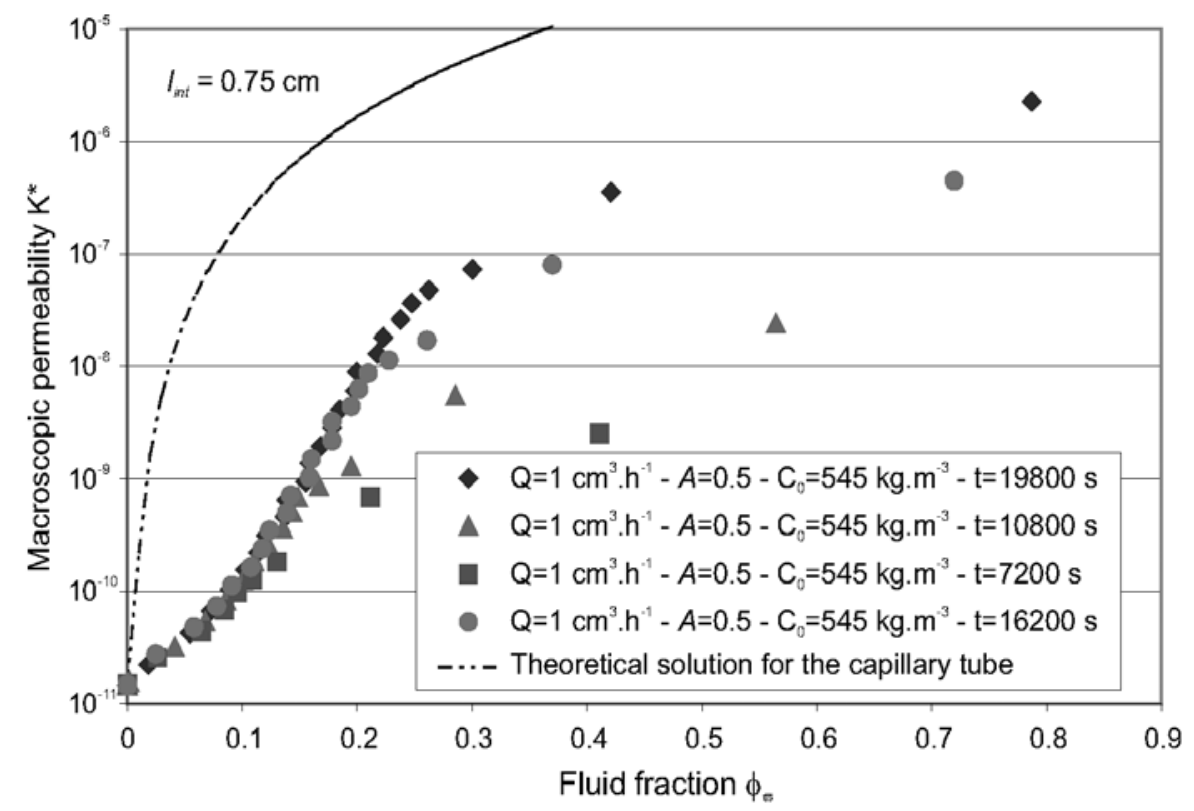

Figure 5-Macroscopic permeability as a function of the fluid fraction (conical regime).

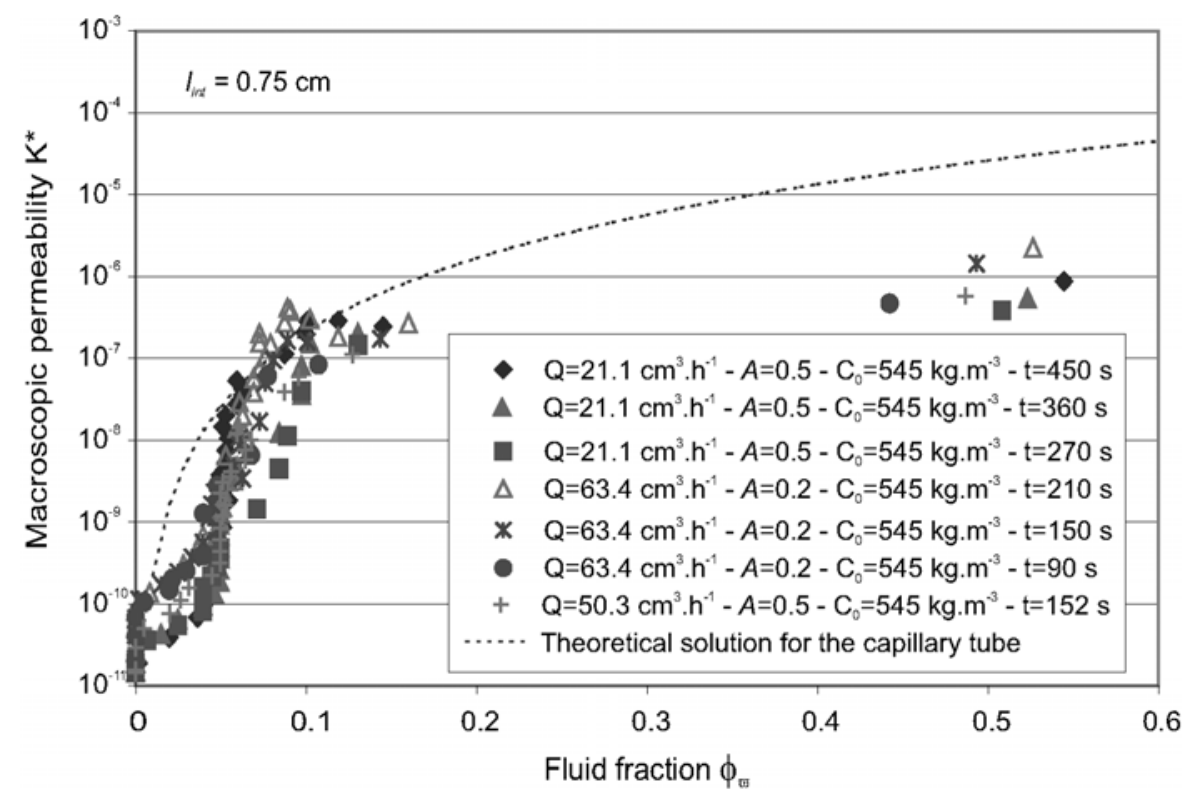

Figure 6 - Macroscopic permeability as a function of the fluid fraction (wormholing regime). 
regime, a macroscopic relation quasi independent of the time evolution exists and can be expressed as

$$
K^{*}=f\left(\phi_{\varpi}\right)
$$

The influence of the integration length has also been investigated. Figure 7 shows the $\mathbf{K}^{*}-\phi_{\varpi}$ relationship, for various values of $l_{\text {int }}$. This influence is $n e g$ ligible, as long as the interval length remains strictly smaller than the wormhole length $L_{w}$.

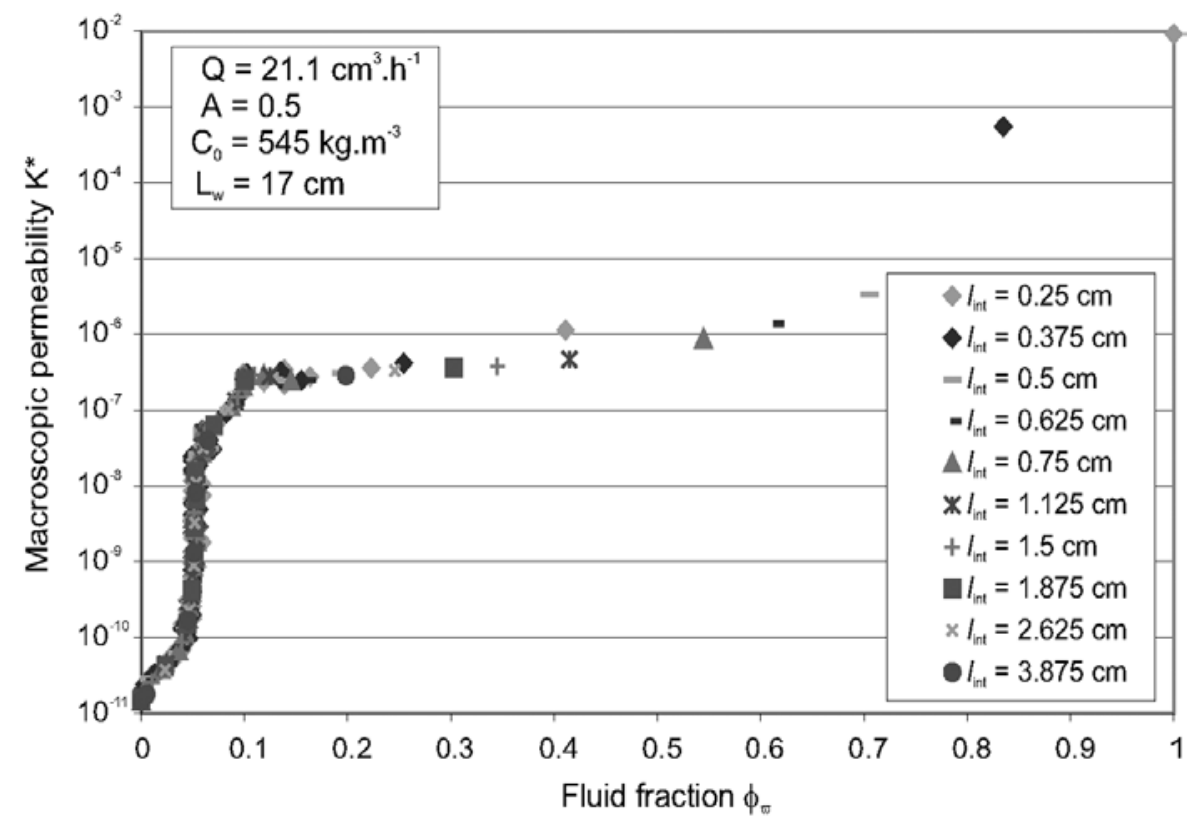

Figure 7 -Effect of the variation of the integration interval length (wormholing regime).

\subsubsection{Ramified regime}

For the ramified regime, at last, Fig. 8 shows the permeability evolution as a function of the fluid fraction for different injection rates, different values of the mass transfer coefficient, and different times. It is obvious that a $K^{*}-\phi_{\varpi}$ relation does not seem to be a very accurate representation. This is due to the impact of the spreading of the dissolution front. In fact, there are now three distinct zones in the domain: a fluid zone, a zone corresponding to the 
initial porous medium, and a transient zone with a porosity gradient due to the conditions of local non-equilibrium. The macroscopic permeability $K^{*}$ can increase although the fluid fraction value is still 0 (cf. triangles $\boldsymbol{\Delta}$ in Fig. 8). Therefore, $\phi_{\varpi}$ does not seem to be convenient to correctly represent the geometry of the domain. While our above discussion suggests that $\phi_{\varpi}$ and $\varepsilon_{\beta}^{*}$ should be used independently, the results interpreted in terms of $\varepsilon_{\beta}^{*}$ only lies close to a single curve, Fig. 9. If confirmed, the core-scale relation could be written as follows

$$
K^{*}=f\left(\varepsilon_{\beta}^{*}\right)
$$

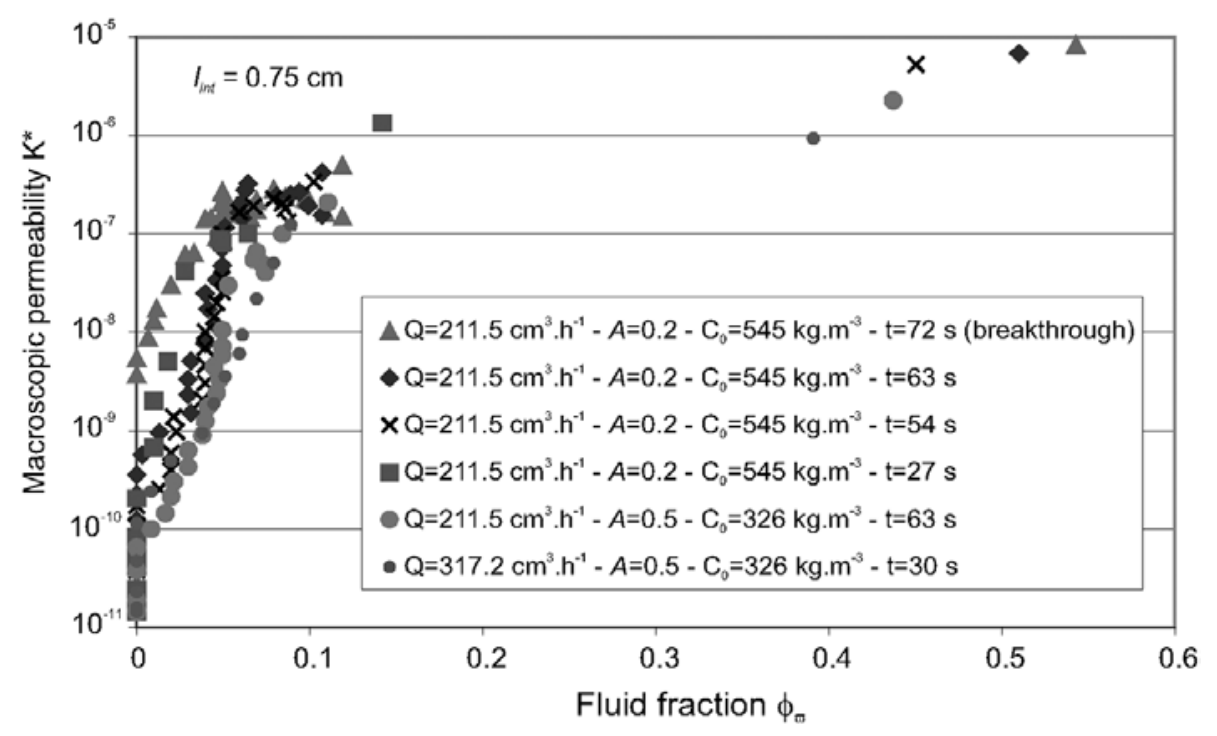

Figure 8-Macroscopic permeability as a function of the fluid fraction (ramified regime).

The influence of the length $l_{\text {int }}$ on the calculation of $K^{*}$ has also been verified and the simulations have confirmed that it was negligible provided it is strictly inferior to the wormhole length.

\subsection{Macroscopic mass transfer relationship}

The core-scale mass transfer coefficient $\alpha^{*}$ seems more difficult to estimate. Based on the relation at the Darcy-scale $\alpha=f\left(P e_{\text {cell }}, \varepsilon_{\beta}\right)$ and from the closure problem form, we start by assuming that the coefficient $\alpha^{*}$ depends on the Peclet 


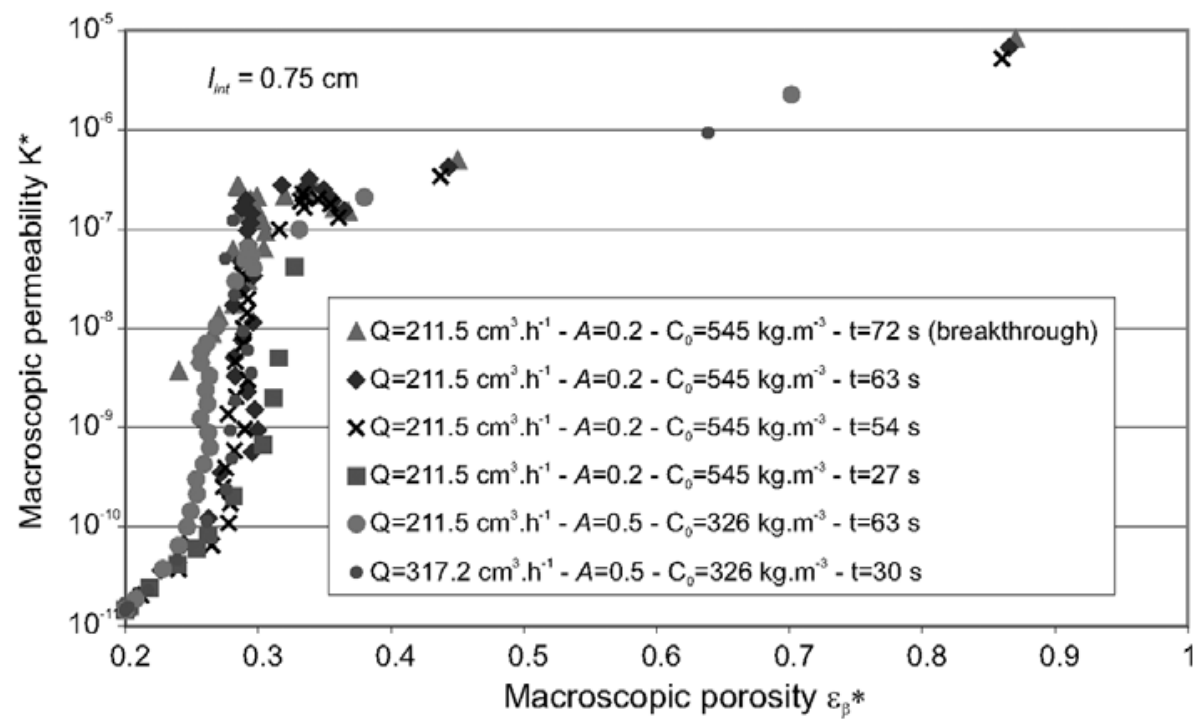

Figure 9-Macroscopic permeability as a function of the macroscopic porosity (ramified regime).

number $P e$ at the core-scale and the fluid fraction $\phi_{\varpi}$ (or the core-scale porosity $\varepsilon_{\beta}^{*}$ for the local non-equilibrium dissolution regime). Moreover, we assume here that the dependence on the Peclet number is small compared with the dependence versus $\phi_{\varpi}\left(\right.$ or $\left.\varepsilon_{\beta}^{*}\right)$. We will come back later on this assumption.

We have represented in Fig. 10 the evolution of the core-scale mass transfer coefficient $\alpha^{*}$ into the core as a function of the core-scale porosity $\varepsilon_{\beta}^{*}$ for different dissolution times and different injection rates within the conical, wormholing and ramified regimes. This figure shows that the relationship $\alpha^{*}-\varepsilon_{\beta}^{*}$ is not as evident as the relationship $K^{*}-\varepsilon_{\beta}^{*}$, although the plot is here in semi-log scale. Even if a relationship seems to emerge in the case of the conical regime, we can remark the same phenomenon of instationarity observed for the $K^{*}-\varepsilon_{\beta}^{*}$ relationship. At $\varepsilon_{\beta}^{*}=0.2$, initial porosity of the medium, we recover the mass transfer coefficient value at the Darcy-scale. When the fluid fraction (resp. the core-scale porosity) increases, the core-scale mass transfer coefficient decreases to pass by a plateau before going to 0 when $\varepsilon_{\beta}^{*} \rightarrow 1$. The value depends on the dissolution regime. In fact, if $\alpha$ is proportional to $1 / l_{\beta}^{2}$ where $l_{\beta}$ represents the characteristic length at the pore-scale (here $l_{\beta}=300 \mu \mathrm{m}$ ), $\alpha^{*}$ is propor- 


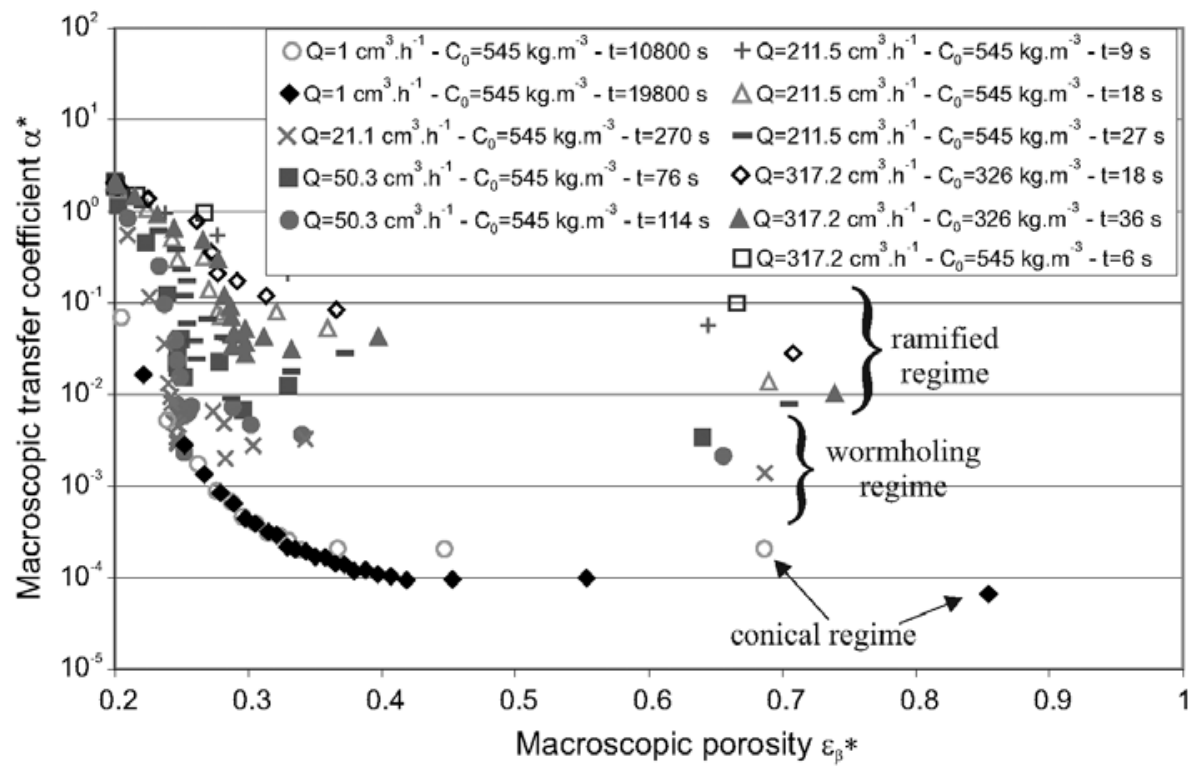

Figure 10 - Macroscopic mass transfer coefficient as a function of the macroscopic porosity $\varepsilon_{\beta}^{*}$.

tional to $1 / l_{\varpi}^{2}$ where $l_{\varpi}$ is the wormhole width. Therefore, as the wormhole width decreases from the conical regime to the wormholing regime, and from the wormholing regime to the ramified regime, although it is more difficult to define the limit of a wormhole in local non-equilibrium dissolution, the value of the core-scale parameter $\alpha^{*}$ decreases in the same way. Thus, when the macroscopic porosity $\varepsilon_{\beta}^{*}$ increases, the core-scale mass transfer coefficient decreases of a factor $\left(l_{\beta} / l_{\varpi}\right)^{2}$ versus its initial value at $\varepsilon_{\beta}^{*}=0.2$.

The uncertainty about the relationship for $\alpha^{*}$ raises a major problem: like at the lower-scale, several numerical simulations confirmed that the propagation velocity of the wormholes predicted by our core-scale model is strongly sensitive to the value of this coefficient, especially in the zone at the end of the wormhole $\left(\varepsilon_{\beta}^{*}=0.3\right)$. Based on the assumption that this uncertainty is linked with the multitude of channels created at the short times at the core inlet, we decided to eliminate from the porosity and concentration fields the wormholes that do no longer develop, and to only consider the influence of the dominant wormhole (this will be referred to as the dominant wormhole assumption). The 
(a)
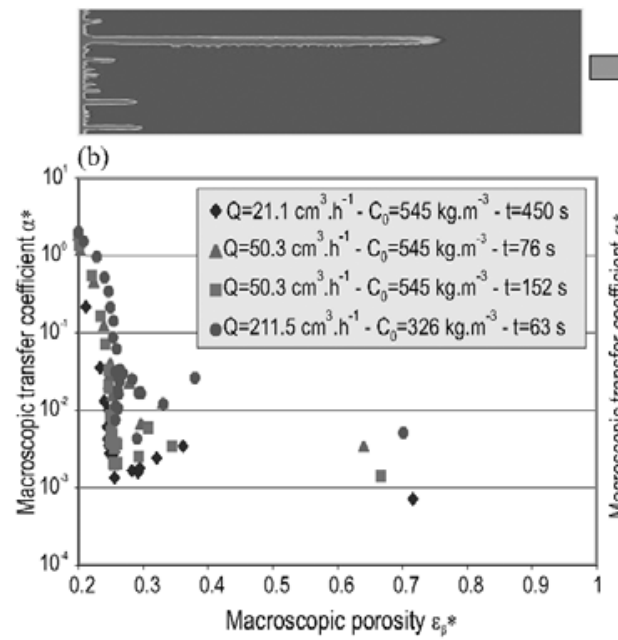

(c)

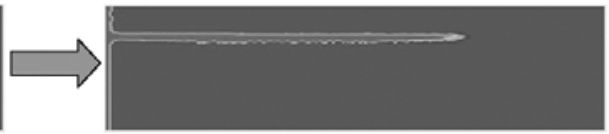

(d)

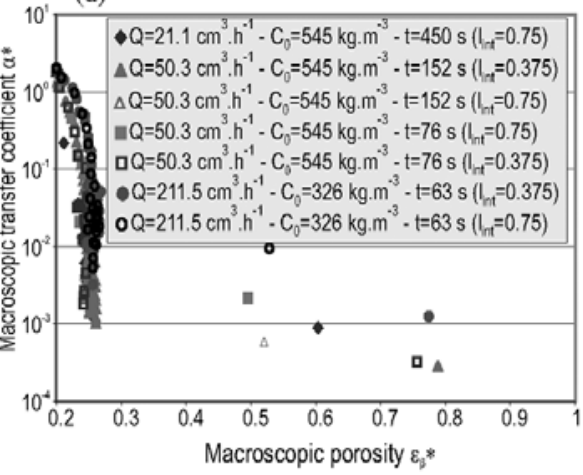

Figure $11-(\mathrm{a})$ porosity field and (b) $\alpha^{*}-\varepsilon_{\beta}^{*}$ correlation in wormholing regime before the dominant wormhole assumption; (c) porosity field and (d) $\alpha^{*}-\varepsilon_{\beta}^{*}$ correlation in wormholing regime after the dominant wormhole assumption.

interest of using this assumption is confirmed by Fig. 11. The variation of the integration interval length has always a negligible influence on the correlation. An interesting parallel can be made between this assumption and the quasistationarity condition often used for solving the closure problems. With this last assumption, non-local effects in time and space are eliminated. In the same way, neglecting these wormholes allows to neglect the spatio-temporal variations of the deviations, and, consequently, the theory is valid only for some long times and far enough from the core inlet.

A zoom around the zone where the mass transfer coefficient strongly decreases, and which contains the most important part of the dissolution physics, is represented in Fig. 12. This shows a non negligible dependence of the $\alpha^{*}$ coefficient versus the injection velocity, i.e., the Peclet number. It is therefore necessary to take into account both the fluid fraction (or the porosity $\varepsilon_{\beta}^{*}$ ) and the injection Peclet number for the development of the correlations for the corescale mass transfer coefficient. The log-normal representation in Fig. 12 suggests the use of correlations for $\alpha^{*}$ with an exponential dependence with $\varepsilon_{\beta}^{*}$, the magnitude varying as a function of the injection Peclet number. 


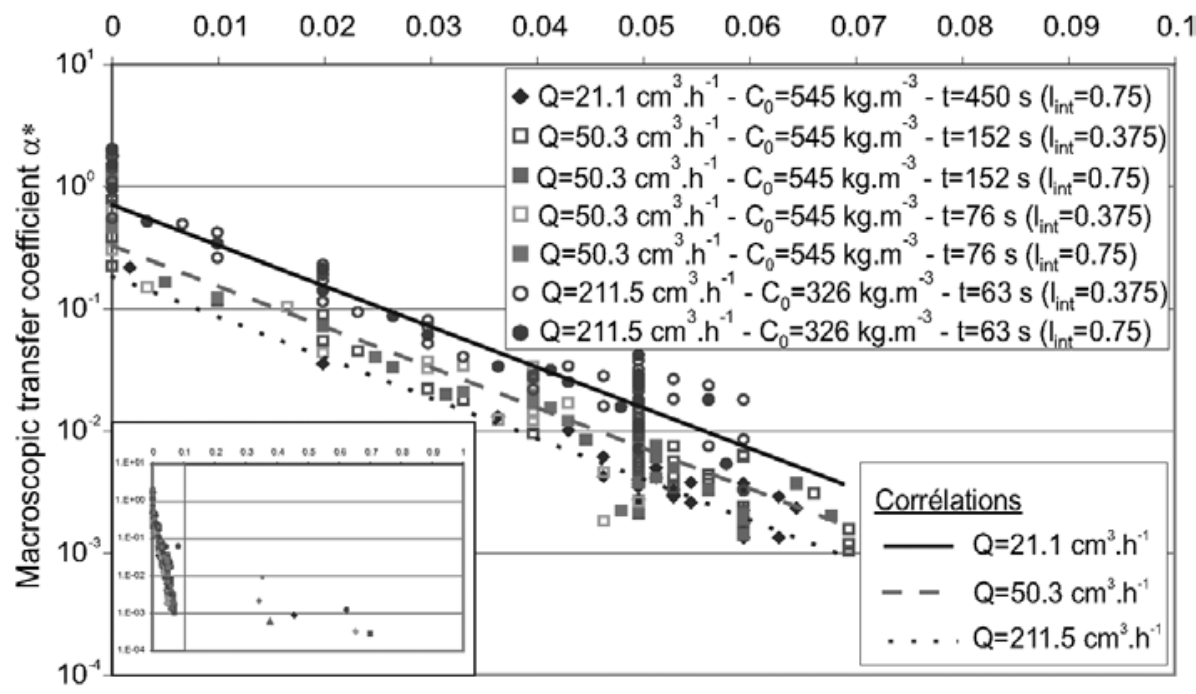

Fluid fraction $\phi_{\infty}$

Figure 12 - Expression of correlations $\alpha^{*}=f\left(P e, \phi_{\varpi}\right)$.

As a conclusion, several important results can be extracted from the development of these correlations:

- In the conical regime, effective coefficients cannot be uncoupled from the dissolution history.

- Simple correlations for the mass transfer coefficient remains difficult to be evaluated numerically. This represents an important problem for the development of a macroscopic model at the core-scale, while it is not clear at this point what is the impact of errors made in determining these correlations.

- The obtained correlations do not depend on the length of the integration interval $l_{i n t}$, within the range of parameters explored.

\section{Core-scale 1D model}

From the system of equations obtained at the core-scale and the mass transfer and permeability correlations provided by the numerical simulations at the Darcy-scale, two 1D core-scale models can be used to verify the validity of 
these representations. The one-medium or one-equation local non-equilibrium model corresponds to the coupling between the flows equations, Eqs. (3) and (4), and the transport and dissolution equations, Eqs. (8) and (9). The development of the two-medium or two-equation local equilibrium model, based on the reaction-transport equations presented previously, Eqs. (6) and (7), needs the introduction of an additional assumption. In fact, this model requires the knowledge of the regional average velocities $\mathbf{V}_{\varpi}^{*}$ and $\mathbf{V}_{\eta}^{*}$, i.e., solving of the regional core-scale Darcy equations written as

$$
\begin{aligned}
\nabla \cdot \mathbf{V}_{\varpi}^{*} & =0 \\
\mathbf{V}_{\varpi}^{*} & =-\frac{1}{\mu} \mathbf{K}_{\varpi}^{*} \cdot\left(\nabla P_{\varpi}^{*}-\rho_{\beta} \mathbf{g}\right) \quad \text { in the } \varpi-\text { phase }
\end{aligned}
$$

and

$$
\begin{aligned}
\nabla \cdot \mathbf{V}_{\eta}^{*} & =0 \\
\mathbf{V}_{\eta}^{*} & =-\frac{1}{\mu} \mathbf{K}_{\eta}^{*} \cdot\left(\nabla P_{\eta}^{*}-\rho_{\beta} \mathbf{g}\right) \quad \text { in the } \eta-\text { phase }
\end{aligned}
$$

where $\mathbf{K}_{\omega}^{*}$ and $\mathbf{K}_{\eta}^{*}$ represent the regional effective permeability tensors [8]. The two average velocities are linked with the following relation:

$$
\begin{aligned}
\mathbf{V}_{\beta}^{*} & =\mathbf{V}_{\varpi}^{*}+\mathbf{V}_{\eta}^{*} \\
& =\phi_{\varpi} \mathbf{U}_{\varpi}^{*}+\left(1-\phi_{\varpi}\right) \mathbf{U}_{\eta}^{*}
\end{aligned}
$$

The intrinsic average velocity in the porous medium $\mathbf{U}_{\eta}^{*}$ being negligible versus the velocity in the fluid region $\mathbf{U}_{\varpi}^{*}$, we can assume

$$
\mathbf{V}_{\beta}^{*} \approx \mathbf{V}_{\varpi}^{*}
$$

which allows us to use Eqs. (3) and (4) for the flow part. This approximation raises some difficulties only for some small values of $\phi_{\varpi}$, when the wormhole is not yet developed.

In this section, we test the capacity of these models to describe the core-scale dissolution physics. For this purpose, we compare the predictions given by the 1D averaged models with the simulations performed at the lower scale. Since the large-scale dispersion tensor $\mathbf{D}_{\beta}^{* *}$, which appears in the transport equation, 


\begin{tabular}{|c|c|}
\hline $201 \times 101$ nodes & $A=0.5$ \\
\hline$\rho_{\sigma}=2160 \mathrm{~kg} . \mathrm{m}^{-3}$ & $\mu=1.10^{-3}$ Pa.s \\
\hline$\varepsilon_{\beta}^{*}=0.2\left(\right.$ or $\left.\phi_{\varpi}=10^{-4}\right)$ & $\beta=1.62$ \\
\hline$D=2.10^{-9} \mathrm{~m} . \mathrm{s}^{-1}$ & $K^{*}=1.5 \cdot 10^{-11} \mathrm{~m}^{2}$ \\
\hline
\end{tabular}

Table 1 - Numerical data for constant flow rate injection.

has not been calculated, it has been taken equal to $\phi_{\varpi} D$ (or $\varepsilon_{\beta}^{*} D$ for local nonequilibrium dissolution) in our simulations. This assumption has a small effect on the propagation time because we have focused here on the wormholing and ramified regime, for which the Peclet number is relatively important. The Darcyscale simulations which are used as reference, are obtained from the model described in Golfier et al. [1] and described briefly in Section 2. The local characteristics of the porous medium and its core-scale properties, including the initial values of $\varepsilon_{\beta}^{*}$ (or $\phi_{\varpi}$ ) and $K^{*}$ are given in Table 1 . The correlations used for the evolution of the mass transfer coefficient $\alpha^{*}$ and the effective permeability $K^{*}$ are obtained as previously described.

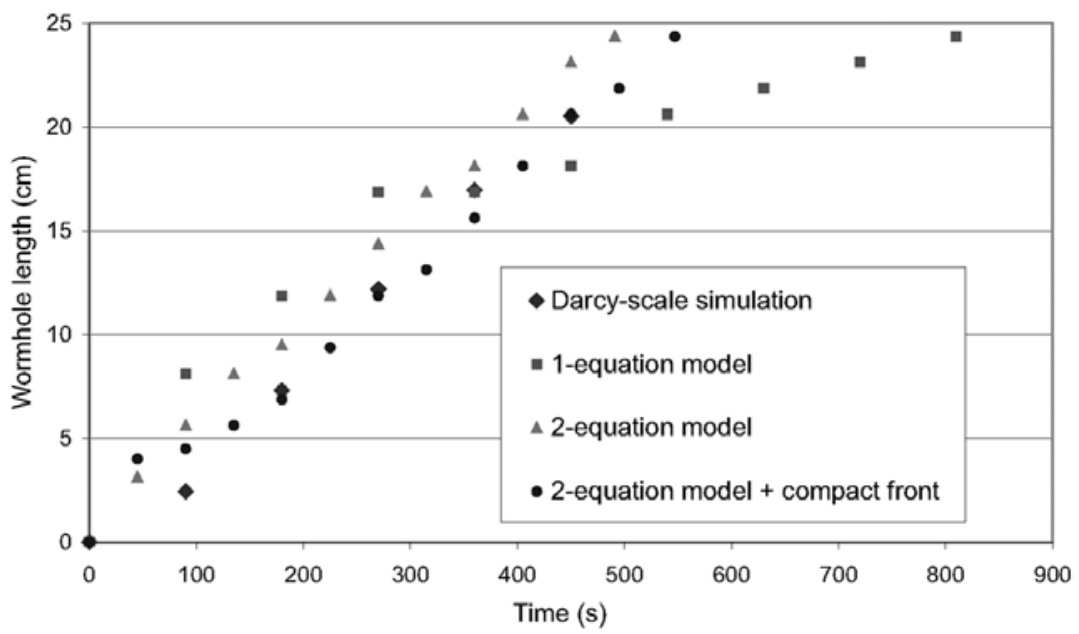

Figure 13 - Comparison of the Darcy-scale model and the core-scale models for $Q=21$ $\mathrm{cm}^{3} \cdot \mathrm{h}^{-1}$ and $C_{0}=545 \mathrm{~kg} \cdot \mathrm{m}^{-3}$.

The comparison of our results for both models with Darcy-scale simulations are illustrated in Fig. 13 and 14 for $Q=21 \mathrm{~cm}^{3} \cdot \mathrm{h}^{-1}-C_{0}=545 \mathrm{~kg} \cdot \mathrm{m}^{-3}$ and 


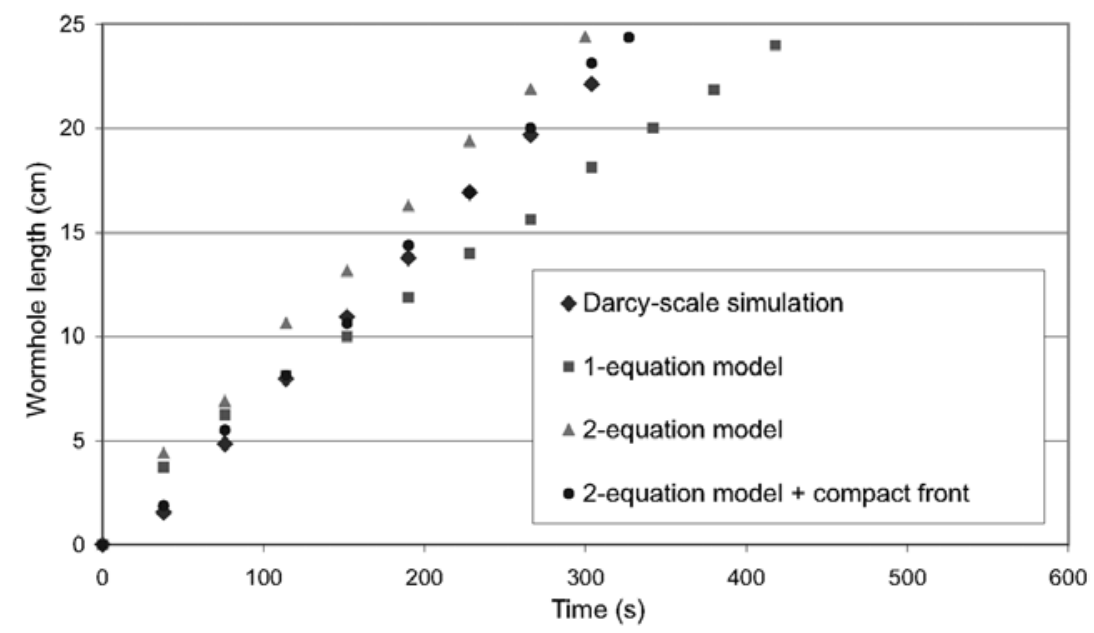

Figure 14 - Comparison of the Darcy-scale model and the core-scale models for $Q=50$ $\mathrm{cm}^{3} \cdot \mathrm{h}^{-1}$ and $C_{0}=326 \mathrm{~kg} \cdot \mathrm{m}^{-3}$.

$Q=50 \mathrm{~cm}^{3} \cdot \mathrm{h}^{-1}-C_{0}=326 \mathrm{~kg} \cdot \mathrm{m}^{-3}$, respectively. It must be emphasized here that the choice of the porosity threshold used to determine the tip of the wormhole is a decisive criterion: it has been fixed from the observation of the porosity fields at $\varepsilon_{\beta}^{*}=0.25$ ( $\phi_{\varpi}=10^{-2}$ for the two-medium model). In the case of the two-equation model, the initial value of $\phi_{\varpi}$ at $t=0 \mathrm{~s}$, theoretically equal to 0 , is fixed at $10^{-4}$ for numerical reasons. The one-equation model does not succeed to correctly reproduce the propagation velocity of the wormhole and overestimates the breakthrough time in both regimes. The wormhole propagation, indeed, is represented in the one-equation model by an increase of $\varepsilon_{\beta}^{*}$ which is a property averaged on all the domain under consideration. For instance, a uniform dissolution of the porous medium and a conical wormhole well-developed could lead to the same value of $\varepsilon_{\beta}^{*}$ in certain conditions. As a consequence, the macroscopic porosity and hence the one-equation model, are not suitable to describe the local behaviour of the wormhole developement within a porous medium. On the contrary, results are more promising for the two-equation model. The propagation velocity is correctly predicted (same slope coefficient), except for the short times where the propagation time is underestimated. This difference can be explained by the fact that the phenomenon of wormhole competition is not taken into account in the calculation of $\alpha^{*}$ (dom- 
inant wormhole assumption). This emphasizes the difficulty to express this coefficient independently of the history. The comparison of the fluid fraction fields obtained, either directly from the core-scale model, or by spatial integration of the Darcy-scale porosity fields, confirms this assumption. One can see in Fig. 15 , that the $\phi_{\varpi}$ value is correctly predicted, except for the inlet of the domain where it is strongly underestimated because the core-scale model does not take into account the multitude of channels which propagate over a few centimeters.

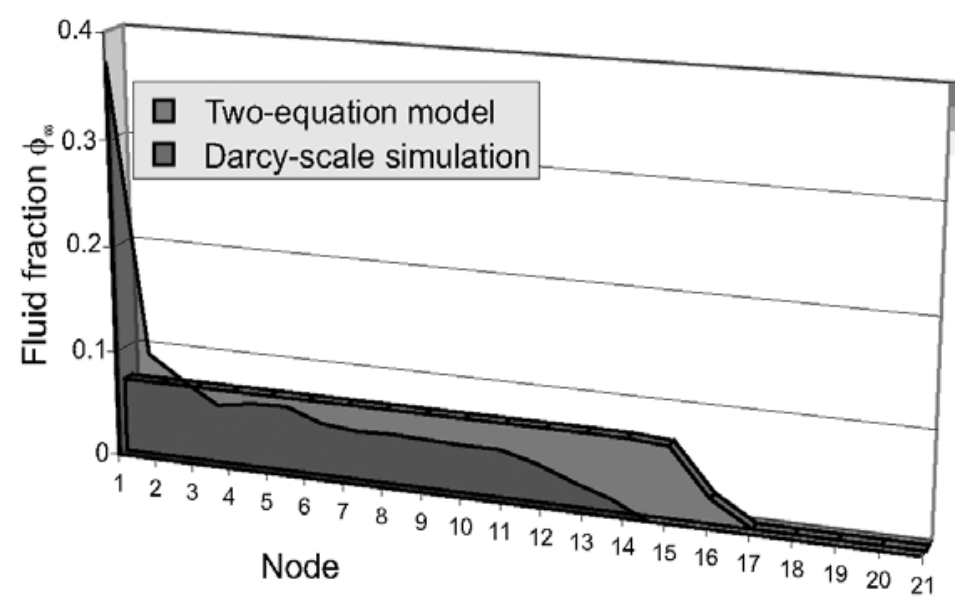

Figure 15 - Fluid fraction values predicted by the Darcy-scale model and the core-scale 2-equation model for $Q=50 \mathrm{~cm}^{3} \cdot \mathrm{h}^{-1}$ and $C_{0}=326 \mathrm{~kg} \cdot \mathrm{m}^{-3}$ at $t=152 \mathrm{~s}$.

These first results suggest that it is impossible to completely eliminate the non-local effects and that a unique correlation for the mass transfer coefficient, independent of $x$ and $t$, does not allow to perfectly reproduce the dissolution phenomenon at the core-scale. In the framework of the proposed core-scale models, several routes can be considered to model these mechanisms and correct the inaccuracy induced by such a representation: (i) taking into account the time effects by the introduction of a different $\alpha^{*}$-correlation at short times such as $\alpha^{*}=\alpha_{\text {compact }}^{*}=$ constant for $t \leqslant t_{0}$, (ii) taking into account the spatial effects by the introduction of a different $\alpha^{*}$-correlation at the inlet of the domain such as $\alpha^{*}=\alpha_{\text {compact }}^{*}=$ constant for $x \leqslant x_{0}$. We have chosen here, from the observation of the fraction fluid values, a "mixed approach" by using a different correlation for $\alpha^{*}$ where the porous medium at the inlet is not dissolved enough, i.e., 
$\alpha^{*}=\alpha_{\text {compact }}^{*}=$ constant for $\phi_{\varpi}^{\text {inlet }} \leqslant \phi_{0}$. Then, the $\alpha_{\text {compact }}^{*}$ variable becomes an additional parameter of the model that may be fitted to "experimental" data. The results of the introduction of this inlet compact dissolution model are illustrated by the curve with the symbols $(\bullet)$ in Fig. 13 and 14 and the impact on the fluid fraction field is presented in Fig. 16.

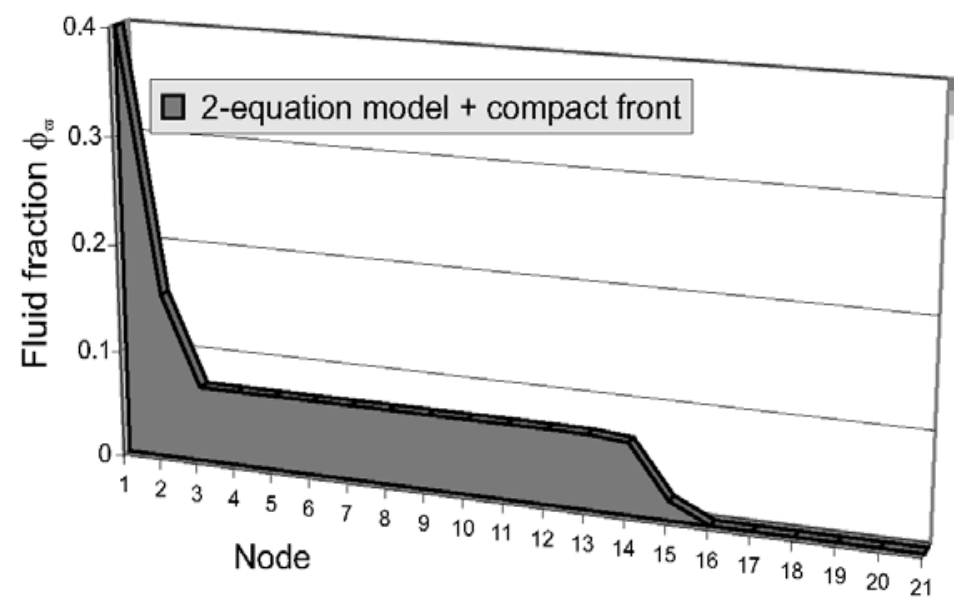

Figure 16 - Fluid fraction values predicted by the core-scale 2-equation model with compact front for $Q=50 \mathrm{~cm}^{3} \cdot \mathrm{h}^{-1}$ and $C_{0}=326 \mathrm{~kg} \cdot \mathrm{m}^{-3}$ at $t=152 \mathrm{~s}$.

These different results confirm that the description of the studied system by a two-medium domain may be an appropriate way for describing the dissolution phenomenon at the core-scale.

\section{Conclusion}

We have determined in this paper the correlations used for the effective coefficients which appear in the two core-scale transport-reaction models developed in Part I [2]. In both cases, the correlations have been directly extracted from the study of Darcy-scale numerical simulations and not from the solution of "closure problems".

Several questions are left open concerning both the form of the macroscopic equations and the correlations used for the effective coefficients. Nevertheless, the results suggest that a two-medium model is a better candidate to describe the physic behaviour of the dissolving system. Furthermore, it seems that the 
effective coefficients cannot completely be uncoupled from the non-local effects and the expressions of their correlations remains non-local functions, i.e., functions of $x$ and $t$. A good estimation of the mass transfer coefficient $\alpha^{*}$ allows however to correctly reproduce the wormhole propagation into the domain.

What are the implications of these results for larger "averaging" volume, i.e., field scale problems? The introduction of a supplementary upscaling to describe the wormholing phenomenon at the large-scale seems difficult. The use of our core-scale model as a near well-bore simulator coupled with a classical field model seems preferable. In fact, the results of our model could be used to calculate the skin effect factor in order to take into account the real conditions around the well within the field model. Therefore, we investigate at this time the ability of core-scale models to correctly reproduce the experimental results being based on accessible experimental data only, i.e., porosity and fluid fraction fields which can be obtained from tomography methods.

\section{REFERENCES}

[1] F. Golfier, C. Zarcone, B. Bazin, R. Lenormand, D. Lasseux and M. Quintard. On the ability of a Darcy-scale model to capture wormhole formation during the dissolution of a porous medium. J. Fluid Mech., 457 (2002), 213-254.

[2] F. Golfier, B. Bazin, R. Lenormand and M. Quintard. Core-scale description of porous media dissolution during acid injection - part I: Theoretical development. Comp. Appl. Math., 23(2-3) (2004), 173-194.

[3] B. Goyeau, T. Benihaddadene, D. Gobin and M. Quintard. Numerical calculation of the permeability in a dendritic mushy zone. Metall. and Mater. Trans. B, 30B (1999), 613-622.

[4] M.L. Hoefner and H.S. Fogler. Pore evolution and channel formation during flow and reaction in porous media. AIChE J., 34(1) (1988), 45-54.

[5] C.M. Marle. Ecoulements monophasiques en milieu poreux. Rev. Inst. Français du Pétrole, 22(10) (1967), 1471-1509.

[6] C. Moyne, S. Didierjean, H.P. Amaral Souto and O.T. Da Silveira. Dispersion thermique en milieu poreux: Modèle à une équation. In Congrès Annuel de la Société Française des Thermiciens, pages 467-472. Elsevier, 1999.

[7] D.R. Poirier. Permeability for flow of interdentritic liquid in columnar-dentritic alloys. Met. Trans., 18B (1987), 245-255.

[8] M. Quintard and S. Whitaker. Transport in chemically and mechanically heterogeneous porous media - II. Large-scale mechanical equilibrium and the regional form of Darcy's law. Adv. in Water Resour., 21 (1998), 617-629. 
[9] M. Quintard, F. Cherblanc and S. Whitaker. Dispersion in heterogeneous porous media: One-equation non-equilibrium model. Transport in Porous Media, 44(1) (2001), 181-203.

[10] S. Whitaker. Diffusion and dispersion in porous media. AIChE J., 13 (1967), 420-427. 